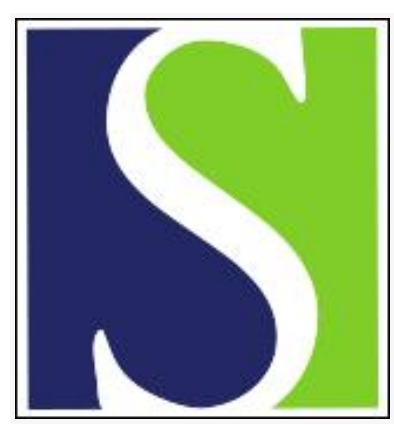

Scand J Work Environ Health 1992;18(2):73-83

https://doi.org/10.5271/sjweh.1598

Issue date: 01 Apr 1992

Relationship of ergonomic stressors to birthweight and gestational age.

by Marbury MC

Affiliation: Minnesota Department of Health, Section of Chronic Disease and Environmental Epidemiology, Minneapolis 55440.

This article in PubMed: www.ncbi.nlm.nih.gov/pubmed/1604276

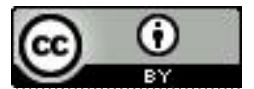




\title{
Relationship of ergonomic stressors to birthweight and gestational age
}

\author{
by Marian C Marbury, ScD 1
}

\begin{abstract}
MARBURY MC. Relationship of ergonomic stressors to birthweight and gestational age. Scand $J$ Work Environ Health 1992;18:73-83. Women in many occupations are exposed to ergonomic stressors (eg, prolonged standing, physical exertion, and long work weeks). After a consideration of the major methodological problems encountered in studying effects of ergonomic stressors on birthweight and gestational age, epidemiologic studies that have addressed this issue are reviewed. No individual ergonomic stressor has been consistently associated with effects on gestational age. However, most studies which have attempted to look at the cumulative effect of several stressors report that women in the highest exposure category are at higher risk of giving birth prematurely. In contrast, data regarding an association with birthweight are more variable, and firm conclusions cannot be drawn. While future studies that assess exposure to ergonomic stressors with a more comprehensive and quantitative approach are needed, current evidence is sufficient to suggest that clinicians should take ergonomic stressors into account in the assessment of their patients' risk for preterm delivery.
\end{abstract}

Key terms: adverse occupational effects, epidemiologic evidence, heavy lifting, physical work, posture, pregnancy, review.

The increase of women of reproductive age in employment in many countries has stimulated scientific investigation of the relationship between work and pregnancy outcome. In addition to studies that have evaluated the reproductive toxicity of specific chemicals or jobs, a growing body of literature has addressed effects of ergonomic stressors.

Ergonomic stressors include factors that are related either to physical exertion (such as heavy or frequent lifting, prolonged standing or sitting, work with heavy machinery, and climbing stairs or ladders) or to work organization (including hours worked, shift work, machine-paced work, and speed of work). Ergonomic stressors may be the most common occupational exposure of women in both traditional and nontraditional occupations. Thus understanding the impact of these stressors should be high priority.

An effect of ergonomic stressors on gestational age and birthweight is biologically plausible. Fuchs \& Stubblefield (1) proposed that adverse effects may be mediated via the sympathetic nervous system and the release of prostaglandins into maternal circulation. A wide variety of factors, including heavy physical work and fatigue, can cause stress. Stress results in the release of catecholamines, which increase blood pressure and uterine irritability and decrease placental function.

\footnotetext{
I Minnesota Department of Health, Section of Chronic Disease and Environmental Epidemiology, Minneapolis, Minnesota, United States.
}

Reprint requests to: Dr MC Marbury, Section of Chronic Disease and Environmental Epidemiology, Minnesota Department of Health, 717 Delaware Street SE, PO Box 9441, Minneapolis, Minnesota 55440, USA.
Uterine irritability causes cervical changes, which then initiate the onset of labor. In addition, decreased placental function decreases progesterone production, and results in an increase in prostaglandin synthesis, which also promotes cervical changes. Catecholamine and prostaglandin levels have also been shown to increase during exercise, with the same effects on uterine contractility.

As studies of ergonomic stress and spontaneous abortion have recently been reviewed (2), I have focused on outcomes of late pregnancy, in particular gestational age and birthweight, in this report. Outcomes such as pregnancy-induced hypertension may also be important and biologically plausible, but the available data are still sparse. The objectives of this paper are to review critically the epidemiologic literature, including common methodological issues and problems, to summarize the available information, and to discuss future research directions.

\section{Methodological issues}

\section{Exposure assessment}

In studies of ergonomic stressors, job titles are often used as indirect measures of exposure in the absence of information on ergonomic factors. This practice is particularly common in the secondary analysis of data sets collected primarily for other nonoccupational purposes. Using job title inevitably results in some degree of misclassification of exposure status. For example, nurses perform a wide variety of jobs, some of which are physically stressful and some of which are not. Such misclassification, if it is random with respect to 
outcome, reduces the apparent association between exposure and outcome. Job title can also be correlated with other characteristics that affect pregnancy outcome, such as socioeconomic status, and thereby introduce the possibility of confounding.

Direct assessment of exposure can be subjective or objective. Subjective assessment usually entails the administration of standardized questionnaires to study participants concerning the extent to which they are exposed to a variety of ergonomic stressors. Questions can be asked regarding the amount of lifting the job entails, the number of hours spent standing, and so forth. The validity of the information depends on the participants' ability to recall and quantify accurately factors which are likely to vary over time. If the questionnaire is administered after delivery, as is common, recall bias can also occur. Having participants keep daily logs of activities is likely to provide more accurate data than a questionnaire, provided that the logs are kept for a period long enough to capture the variability of the work tasks. Such an approach has not been attempted in studies of ergonomic stressors and pregnancy outcomes.

An objective measure of exposure might entail trained ergonomists' directly observing and perhaps videotaping work practices. While this approach has been used in studies of repetitive motion trauma, it is extremely time intensive, and its application in studies of a reasonable size would be difficult. Nonetheless, it might be useful in the validation of a questionnaire for some subset of study participants. The development of methods to measure metabolic activity suitable for large population studies would also provide an objective quantitative measure of exposure.

Failure to consider exposure to ergonomic stressors outside the workplace is another common inadequacy in exposure characterization. For example, the amount and type of recreational exercise, the amount of housework and the help available in performing it, and the number and ages of children at home may all substantially modify the degree of ergonomic stress experienced by pregnant women.

The choice of a method for exposure characterization is often based on feasibility rather than validity. The further removed the exposure variable is conceptually from the actual exposure, the greater the opportunity for misclassification. Furthermore, if the exposure variable has been vaguely defined, then specific ameliorative measures are not obvious.

\section{Definition of outcome}

The definition and ascertainment of birthweight and gestational age can vary among studies, and this variation must be considered when studies are compared. While data on birthweight are relatively straightforward to collect and usually considered of high quality, analyses of birthweight have differed in two important ways. First, birthweight can be analyzed as a dichotomous ( $\leq 2500 \mathrm{~g}$ versus $\geq 2500 \mathrm{~g}$ ) or a continuous variable. When birthweight is treated as a dichotomous variable, statistical analysis focuses on differences in the proportions of low birthweight babies in two or more exposure groups. The rationale for dichotomization is that morbidity and mortality are associated with birthweight below this cutoff. However, this approach necessitates the use of a larger sample size and will not detect those exposures that may have a more subtle effect on birthweight or an effect on a small number of babies. When birthweight is treated as a continuous variable, the statistical analysis is concerned with differences in mean birthweight between two or more exposure groups. Because such an analysis requires a smaller sample size, it, in general, is preferable.

Second, not all studies have taken into account the effect of gestational age on birthweight. Gestational age is the primary determinant of birthweight. Birthweight can be low either because of premature birth or because of intrauterine growth retardation. This is a crucial distinction as it reflects two different pathologic mechanisms. Consequently, studies which have failed to make this distinction are difficult to interpret.

Estimates of gestational age may be based on the subjects' recall of the dates of the last menstrual cycle, on the physician's estimate, or on ultrasound. Analytically, gestational age has been treated as both a dichotomous and a continuous variable. As a dichotomous variable, preterm birth is usually defined as birth occurring before 37 complete weeks of gestation, counting from the first day of the last menstrual period (3). Similar to birthweight, the rationale for considering prematurity, rather than changes in mean gestational age, is that prematurity is associated with a substantial degree of neonatal morbidity and mortality. Again, however, such a statistical analysis has less power to detect subtle effects than one based on a comparison of the mean gestational ages among exposure groups.

\section{Study design}

Cross-sectional, case-referent, and prospective cohort study designs have all been used to assess the relationship between ergonomic exposures and pregnancy outcomes. In the cross-sectional design, women are interviewed soon after delivery about a wide variety of exposures that occurred before or during pregnancy or both. This design has been widely employed because of the relative ease with which a large number of subjects can be identified and interviewed and because of the number of late pregnancy outcomes that can be evaluated. While the feasibility of this type of study has made it particularly attractive, several important limitations need to be recognized. Since data on exposures are gathered retrospectively after the birth, either random misclassification or recall bias can occur. In addition, either the exposure of interest must 
be fairly common in the general population or the study population must be large. Finally, the temporal sequence between exposure and outcome cannot always be identified. For example, if a woman who has been working strenuously experiences difficulties with her pregnancy, she may well be advised to leave her job prior to delivery. Thus, if the exposure variable is duration of work during pregnancy, those who have worked to term will have more favorable outcomes than those who left work earlier. In this case, the outcome actually determines the exposure, given how the exposure has been defined. Case-referent studies share these same limitations. However, because the number of subjects is usually much smaller, the amount of information gathered per subject may be larger.

In prospective cohort studies, women are enrolled early in pregnancy and followed through delivery. Information on exposures is usually obtained at enrollment and may or may not be updated during the course of the pregnancy. This design avoids the limitations mentioned earlier. Misclassification can still occur because of the inherent difficulty in trying to characterize variable exposures, but it will not be compounded by recall problems or biased with respect to outcome. All of the pregnancy outcomes can be identified, and determining the temporal sequence between exposure and outcome is straightforward.

Prospective cohort studies have two primary drawbacks, their feasibility and exposure prevalence. If the exposure is common, the cohort study can be conducted on the general population, and patients can be identified at the first prenatal visit at several clinics or practices. However, if the exposure is uncommon in the general population, then investigators must either identify a large exposed subpopulation, say, a particular industry, or screen a very large number of women having first prenatal visits. In the first instance, the subpopulation must be large to provide an adequate number of pregnant women. In the second case, a screening mechanism needs to be established to identify women with the specific exposure of interest. Regardless of how women are identified, a follow-up of these women is necessary. Although the feasibility of follow-up is enhanced by the short time of pregnancy, the logistical effort is still greater than for casereferent and cross-sectional studies.

\section{Confounding factors}

Many factors are known to influence both birthweight and gestational age. For example, parity, social class, maternal stature, preeclampsia, maternal hypertension, and smoking have been associated with low birthweight (4). Maternal age, smoking, social class, and a history of perinatal loss have been associated with preterm delivery (5). Clearly some of these factors are also associated with both employment in general and with specific occupations. For example, women of higher parity are less likely to be employed outside the home (6).

Socioeconomic status is potentially the most serious confounding factor when the relationship between ergonomic stressors and pregnancy outcome is being evaluated. Lower socioeconomic status has been consistently associated with adverse pregnancy outcomes, although the nature of the association is not clear. Even when analyses control for characteristics that are associated with lower socioeconomic status, such as maternal age, parity, or smoking, an independent effect of socioeconomic status on low birthwe ght and preterm delivery is still apparent. There is also a strong association between socioeconomic status and the probability of employment in physically stressful work in that lower paying jobs are much more likely to be physically demanding. In addition, socioeconomic status is a complex concept which is probably not adequately captured by individual variables such as the mother's educational level or family income. Even if socioeconomic status is correctly measured, multiple regression coefficients will not be accurate if socioeconomic status is highly correlated with physical stress. This problem can be dealt with most directly if the population is restricted so that all of the subjects are of the same socioeconomic status or there is stratification by socioeconomic status in the analysis. The latter approach, if there are sufficient numbers in all strata, would permit effect modification by social class to be evaluated. It is possible that the effect of ergonomic stress on birth outcome varies by socioeconomic status and, specifically, that those who are more economically disadvantaged may be more susceptible to the adverse influences of ergonomic stress.

Psychological stress is another potentially important confounding factor. It may have the same physiological effect on pregnancy as ergonomic stressors and may also be highly correlated with these stressors. Some studies have attempted to address the relationship between job-related psychological stress and health and between psychological stress from other sources and pregnancy outcome. As methods of characterizing psychological stress are improved, they should be incorporated into studies of ergonomic stressors.

\section{Studies of employment and pregnancy out- come}

Research done in Great Britain in the 1950s suggested that employment outside the home during pregnancy resulted in an increase in the incidence of premature birth (7-9) and stillbirth (8). More contemporary studies from Israel (10), France (11), Great Britain (12), Australia (13), and the United States $(6,14)$ have found that the pregnancy outcomes of employed women are either as favorable or more favorable than the out- 
comes of women who are not employed. Several explanations have been suggested for this favorable association, including the socioeconomic advantages of a household with two wage earners, increased access to medical care for employed women, and the superior health status necessary for employment relative to that of the general population.

An analysis of data from the "Collaborative Perinatal Project"' by Naeye \& Peters (15) provides the one exception to the generally favorable association between employment and pregnancy outcome. Although the analysis was performed in the 1980 s, the data were collected from 1959 to 1966 . In this study, job title was used to classify women into one of the following three categories: not employed outside the home, employed in a sedentary job, and employed in a standing job. The authors reported that birthweight was 150 to $400 \mathrm{~g}$ less for women employed outside the home after 28 weeks of gestation, except for those who were primiparous and worked in sedentary jobs. While this study has been cited as providing evidence that employment outside the home may have a deleterious effect on fetal health, the failure of the analysis to control simultaneously for multiple confounding factors, the noncontemporary nature of the sample, and the inconsistency of the results with other studies cast doubt on the generalizability of the results to today's work force.

Nonetheless, the question remains of whether leaving work at some point prior to pregnancy might be beneficial. While there is limited evidence to suggest that this practice is advantageous in developing countries where work conditions are more arduous (16), the question has not been appropriately addressed in studies from developed countries. This question cannot be answered simply by examining the relationship between outcome and the duration of employment during pregnancy since women can leave work prior to delivery either by choice or because of pregnancy complications. For example, one study reported that women who worked to term had more favorable outcomes than those who left work between the first and eighth month of pregnancy (6). Prospective studies in which date of and reasons for termination are carefully documented will be essential in resolving this issue.

\section{Studies of ergonomic stressors and late outcomes of pregnancy}

The literature on ergonomic stressors and late outcomes of pregnancy literally doubled in 1989-1990, and this increase reflects the increasing recognition of the importance of the issue (table 1). Of the 18 published studies, ergonomic exposures were inferred from job title in six. Because of the inherent limitations in inferring exposure from job title, these studies are discussed separately.

\section{Studies using job title to categorize exposure}

As described previously, Naeye \& Peters (15) used job title to place women in one of three categories. The mean birthweight, not controlled for gestational age, was lower for women who worked outside the home after the 28th week of gestation, particularly for women employed in standing positions. The frequency of placental infarcts was five times higher among women who worked in standing positions and continued to work after the 37th week in comparison with those who left work before the 33rd week. No effect on gestational age was seen.

These findings were not confirmed in studies using somewhat different classification schemes $(14,20)$. Meyer \& Daling (20) performed a case-referent study of babies with low birthweights determined from the birth certificates. On the basis of job title women were classified as not employed outside the home, in jobs involving sitting more than $75 \%$ of the time, in mixed jobs involving standing and sitting between $25 \%$ and $75 \%$ of the time, in standing jobs, and in very active jobs that entailed physically strenuous work more than $25 \%$ of the time. No relationship between low birthweight and activity level was found. Zuckerman and his colleagues (14) constructed different categories from the job title (ie, not employed outside the home, working in a standing position during all or part of the third trimester, and any other work history, including women who worked at jobs involving standing during the first or second trimester. No associations with gestational age or birthweight were seen.

Teitelman and her co-workers (21), in a prospective cohort study of 1206 employed women, defined the following three categories of activity level based on job title: sedentary, standing, and active. Active jobs were defined as those involving continuous or intermittent walking with an active range of motion, such as janitors and physicians. The relative risk (RR) for preterm birth was 2.7 for women in standing jobs when compared with women in active jobs, and their infants' mean gestational age was $2.5 \mathrm{~d}$ shorter. Apparent differences in the low birthweight rate were not statistically significant when confounding was controlled.

Two studies linked job title with other sources of information to categorize exposure. Homer et al (22), in an analysis of data from the "National Longitudinal Survey of Labor Market Experience Youth Cohort," linked the job title of 772 women to a job characteristics scoring system developed by Robert Karasek. The physical exertion variable in this system is based on the question "Does your job require lots of physical effort?," as answered on a four-point scale. This question was used to categorize job titles into four quartiles of physical exertion, which were later collapsed into two categories. Women in the highest physical exertion category were at higher risk of having a preterm, low birthweight baby $(R R=5.1)$, a low birthweight baby $(R R=2.7)$, a preterm infant $(R R=2.0)$, 
Table 1. Studies of ergonomic stressors and outcomes of late pregnancy. (PTB = preterm birth, LBW =low birthweight, SGA = small for gestational age, $\mathrm{OR}=$ odds ratio, $\mathrm{RR}=$ relative risk)

\begin{tabular}{|c|c|c|c|c|}
\hline $\begin{array}{l}\text { Location and } \\
\text { year }\end{array}$ & Study designa & Ergonomic stressors & Effects of birthweight & $\begin{array}{l}\text { Effects on gestational } \\
\text { age }^{\mathrm{b}}\end{array}$ \\
\hline
\end{tabular}

Job title used to infer exposure

United States

$1959-1962(15)$

Cross-sectional (7722)

United States

1985 (20)

United States

1986 (14)

United States

1990 (21)

United States

1990 (22)

United States

1990 (23)

Case-referent (2911

cases; 2911 referents)

Cross-sectional (1507)

Prospective cohort

Cross-sectional (772)

Case-referent $(604$ cases, 6070 referents)
Employment outside home, standing

Activity level

Employment outside home, standing

Posture: standing, sedentary, active

High exertion

Physical demand

Exposure assessed through questionnaire

United States

1983 (17)

Case-referent (185 cases, 313 referents)

France 1984 (24)

Cross-sectional (1928)

France 1985 (26) Cross-sectional (621)

France $1987(27) \quad$ Cross-sectional (2387)

Canada $1988(28) \quad$ Cross-sectiona! (22 761)

Canada $1989(35) \quad$ Cross-sectional (22 761)

Finland 1989 (18) Case-referent (189 cases, 189 referents)

Finland 1989 (29) Cross-sectional (1475)

Sweden

1990 (19)

Guatemala

1990 (30)

Great Britain

1990 (34)

United States 1990 (31)

\section{Prospective (3906) \\ Prospective (15 876)}

Prospective (1507)

Cross-sectional (2228)
Physical activity at work

Occupational fatigue score from 5 sources

Heavy cleaning, load carrying, standing

Load carrying, standing, assembly line work, physically demanding work

Lifting, other effort, standing, assembly line work, environmental conditions, Mamelle's fatigue score

Same as reference 28

Standing, shift work, "heavy loading," environmental conditions

Physical load, based on assessment of energy expenditure

Heavy lifting

Standing, walking, composite activity score

Composite activity measure

Medical residency versus nonresidency, hours worked per week
$150-400 \mathrm{~g}$ decrease in birthweight ${ }^{c}$

No effect

No effect

No effect

OR $=5.1$ for preterm $L B W, O R=2.7$ for LBW

Not assessed

Not assessed

Not assessed

$O R=1.6^{d}$ for $L_{B W}$ for $\geq 2$ exposures

OR for $L^{2 B W^{c}}=2.0^{d}$ for 3-4 exposures, $1.6^{\text {d }}$ for physically demanding work

See reference 35

Lifting heavy weights, shift work associated with decreasing birthweight, no association with fatigue score

Not assessed

Highest load associated with $\mathrm{SGA}, \mathrm{OR}=2.7^{\mathrm{d}}$

No effect

$O R=1.2$ for standing, 1.3 for walking for SGA; high score associated with SGA, SGA/PTB

No effect

No effect
No effect

Not assessed

No effect

OR $=2.7$ if standing $>3 \mathrm{~h}$

$\mathrm{OR}=2.0$

OR $=1.4$ for median demand, 1.8 for high demands

No effect

$\mathrm{OR}=5.1^{\mathrm{d}}$ for highest scores

$O R=3.5^{d}$ for $\geq 2$

exposures

OR $=2.1^{d}$ for $3-4$

exposures

$\mathrm{RR}=1.3$ for lifting weights, working $>45 \mathrm{~h} /$ week; $R R=1.16$ for fatigue score of $\geq 3$

See reference 28

No effect

Highest load associated with gestational age $<280$ days, $\mathrm{OR}=2.2^{\mathrm{d}}$

No effect

$\mathrm{OR}=1.6$ for standing; high score associated with SGA/PTB, nonsignificantly with PTB

Not assessed

Residents at higher risk for preterm labor $\left(O R=2.0^{\mathrm{d}}\right)$ but not PTB

a Numbers in parentheses.

b Odds ratios are calculated for preterm birth unless otherwise specified.

c Birthweight not controlled for gestational age.

d Prevalence odds ratio calculated from the data. 
and a baby of lower mean birthweight $(160 \mathrm{~g})$. These results were substantially the same when the analysis was restricted to primiparous women.

Ramirez and his colleagues (23) examined the association between physical activity and preterm birth in a case-referent study of primigravidas on active duty in the United States Army. Physical activity assessment was based on the physical demand assessment of all military occupational specialties, which in turn was based on the maximum upper body strength required to perform the job under combat conditions. The relative risk for preterm birth increased from 1.4 for women in the medium demand category to 1.8 for those in the very heavy demand category.

Clearly, these studies based on job title are not consistent. Two studies found an effect of ergonomic stressors on birth weight, while three did not, and three found an effect on gestational age, while two did not. All six studies used different and noncomparable methods of categorizing exposure (eg, women who were in Teitelman's "'active" category, and had better outcomes, would have been included in the standing category of the other studies). Resolution of the discrepancies between these studies is not possible, but the limitations of using job title for exposure assessment are apparent.

In the remaining studies described in table 1, data on exposure were obtained by means of an intervieweror self-administered questionnaire at the beginning of prenatal care in prospective studies or soon after delivery in case-referent and cross-sectional studies. Direct comparisons among these studies are hindered by differences in the way exposures were assessed or defined, differences in the definition of the outcomes examined, and differences in the extent to which potentially relevant confounding variables were controlled. Table 2 summarizes the literature on gestational age in relation to the three ergonomic exposure variables most commonly assessed (standing, heavy lifting, and physically strenuous work). Many studies have also considered the combined effect of individual ergonomic stressors in some manner, and these results are in the "composite score" column. Table 3 is similar but considers effects on birthweight. These tables graphically demonstrate the inconsistency of results in the current literature, with the apparent exception of an association between a composite score and preterm birth.

Table 2. Studies of ergonomic stressors and gestational age ${ }^{a}$ (NA $=$ not assessed in this study, $+=$ positive association found between stressor and decreased gestational age, $-=$ no association found between stressor and decreased gestational age)

\begin{tabular}{lccc}
\hline Study & Standing & Heavy lifting & Physically strenuous Composite score \\
\hline Berkowitz et al (17) & - & - & NA \\
Saurel-Cubizolles et al (26) & NA & NA & NA \\
Saurel-Cubizolles \& Kaminski (27) & - & - & - \\
McDonald et al (28) & - & + & - \\
Mamelle et al (24) & + & - & + \\
Mamelle \& Munoz (25) & - & NA & - \\
Hartikainen-Sorri \& Sorri (18) & - & NA & - \\
Launer et al (30) & + & - & NA \\
Ahlborg et al (19) & NA & NA & NA \\
Nurminen et al (29) & NA & NA & NA \\
Klebanoff et al (31) & NA & NA & NA \\
\hline
\end{tabular}

a All studies assessed preterm birth (gestational age $<37$ weeks) except as noted.

$\checkmark$ No association was found between preterm birth and the composite activity score, but the score was associated with premature, small-for-gestational-age babies.

c Dichotomized gestational age into $<280 \mathrm{~d}$ or $\geq 280 \mathrm{~d}$.

Table 3. Studies of ergonomic stressors and birthweight. (NA = not assessed in this study, $+=$ positive association found between stressor and decreased gestational age, $-=$ no association found between stressor and decreased gestational age)

\begin{tabular}{|c|c|c|c|c|}
\hline Study & Standing & Heavy lifting & Physically strenuous & Composite score \\
\hline Saurel-Cubizolles et al $(26)^{a, b}$ & - & NA & NA & - \\
\hline Saurel-Cubizolles \& Kaminski (27) & - & - & + & + \\
\hline Launer et al $(30)^{c}$ & + & NA & NA & + \\
\hline Ahlborg et al $(19)^{d}$ & NA & - & NA & NA \\
\hline Nurminen et al $(29)^{c}$ & NA & NA & NA & + \\
\hline Armstrong et al $(35)^{d}$ & NA & + & NA & - \\
\hline Rabkin et al $(34)^{d}$ & - & NA & NA & - \\
\hline Klebanoff et al $(31)^{c, d}$ & NA & NA & - & NA \\
\hline
\end{tabular}

a Outcome was low birthweight ( $\leq 2500 \mathrm{~g}$ ).

b Did not control for gestational age.

c Outcome was small for gestational age ( $\leq 10$ th percentile weight, $\geq 37$ weeks age). Launer also examined preterm, small for gestational age ( $\leq 10$ th percentile weight, $<37$ weeks age).

d Outcome was mean birhtweight. 


\section{Effects on gestational age}

Mamelle and her colleagues (24) conducted one of the earliest investigations to use questionnaires for assessing exposure to ergonomic stressors. Women who had been employed during pregnancy $(\mathrm{N}=1928)$ were interviewed immediately after delivery about life-style and occupational activity during pregnancy. The occupational data were used to define the following five sources of fatigue: posture (sitting or standing), work on an industrial machine, physical exertion, mental stress, and environment. Each source was scored either zero or one. Some sources were more clearly defined conceptually than others. For example, posture received a score of one if the work involved standing more than $3 \mathrm{~h}$ a day. In contrast, environment received a score of one if the job involved either manipulation of chemical substances or if two of three elements (significant noise level, cold temperature, or very wet atmosphere) were present. Each source was found to be significantly associated with the risk of a premature birth when considered individually. However, when considered simultaneously, mental stress and environment emerged as the two most important factors. When the individual sources were combined into an occupational fatigue score, the proportion of women having a preterm birth rose from 2.3 to $11.1 \%$ as the score increased from 0 to 5 . Women who worked more than $40 \mathrm{~h}$ a week and had a high fatigue score had the highest prematurity rate $(16.7 \%)$.

The investigators also examined the interaction between "medical factors" and physical stress. They defined "medical factors" as the existence of a previous premature delivery, spontaneous abortion, stillbirth, or perinatal death or as pathology during the first five months of pregnancy which might or might not be related to prematurity. In a comparison with the women who had no medical factor or work-related physical stress, the relative risk of preterm delivery was 1.4 for the women who only had a medical factor, 2.1 for the women with only physical stress, and 2.8 for the women with both.

To assess the reliability of the occupational fatigue score, Mamelle \& Munoz (25) conducted a case-referent study of 200 women who delivered before 37 weeks and 400 women who delivered at term. Their initial findings were partially confirmed. Posture and physical exertion were not individually related to preterm birth, environment was related but not significantly, and work on a machine and mental stress were significantly related. [In their paper the numbers are transposed in table 1 , but the interpretation in the text is accurate (N Mamelle, personal communication).] The cumulative fatigue score was again related to the risk of preterm birth, women scoring 1 or 2 having a moderately increased risk [odds ratio (OR) 1.5] and women scoring 3 or higher having the highest risk (OR 1.9) in comparison with women with scores of 0 .

Other investigators have also used a cumulative score approach in studies of ergonomic stress and preterm birth. Saurel-Cubizolles and her co-workers (26) studied 621 women who worked in hospitals. Questionnaires were administered by hospital personnel to women returning to work at the end of postnatal leave. The women who had worked less than 13 weeks during pregnancy or whose postnatal leave extended beyond 36 weeks were excluded from the study. Three work conditions were combined into a composite score (ie, heavy cleaning tasks, carrying heavy loads, and prolonged standing). In the overall sample, the preterm delivery rate was $6 \%$ for the women with a score of zero, $5 \%$ for those with a score of one, $18 \%$ for those with a score of two, and $30 \%$ for those with a score of three. The association between the composite score and preterm birth was especially strong for the ancillary staff responsible for housekeeping.

In a later study of $\mathbf{2 3 8 7}$ women employed during the first trimester of pregnancy, Saurel-Cubizolles \& Kaminski (27) examined the effects of prolonged standing, carrying heavy loads, assembly-line work, and physically demanding work both individually and combined into a cumulative score. Only assembly-line work was individually associated with an increased risk of preterm birth, but again the cumulative score was strongly associated. The proportion of preterm births was $4 \%$ for those with a score of zero, $5.1 \%$ for those with a score of one or two, and $8.2 \%$ for those with a score of three or higher. The women were divided into two occupational groups (ie, production, service, and shop workers and professional, administrative, and clerical workers). The relationship between the cumulative score and preterm birth was apparent in the first group, but the latter contained only 39 women with scores of three or four, and none of these women had a preterm birth.

McDonald and her colleagues (28), in one of a series of reports from the Montreal survey, also examined the effects of both individual stressors and a composite score on preterm birth. The Montreal survey is particularly informative because of its size ( 56067 women were interviewed), its clearer definition of the ergonomic exposure variables, and its careful control of potentially confounding variables. In this particular paper (28), results were reported for 22761 women who were employed for $\geq 30 \mathrm{~h}$ at conception and continued to work under the same conditions. The ergonomic exposures considered in this study included lifting heavy weights more than 14 times a day, other physical effort, standing more than $7 \mathrm{~h}$ a day, assembly-line work, and environmental conditions (noise, vibration, and extremes of temperature). The only ergonomic stressors that demonstrated an individual relationship with preterm birth overall were working $\geq 45 \mathrm{~h}$ a week and lifting heavy weights, although the latter association was not present for women who worked more than 28 weeks. The investigators also constructed an occupational fatigue score using the same criteria as Mamelle, but with the caution that there may have been differences in the degree of physi- 
cal effort and lifting. The fatigue score demonstrated a statistically significant association with preterm birth, but to a less degree than in Mamelle's study. The relative risk was 1.07 for the women with a score of two and 1.16 for those with a score of three or more.

Other investigators have used different approaches in measuring the cumulative effect of multiple sources of ergonomic stress. Nurminen and her co-workers (29) evaluated the physical load of each woman's job according to a standardized method of assessing energy expenditure. Each woman was assigned a major activity level and, if there were clear variations in the load, a minor activity level. These levels were then combined in a time-weighted average or mean level, and the mean activity levels were divided into four categories. Short-term loads, defined as occasional loads that exceeded the major or minor activity level, were also assessed. Since too few women delivered prematurely to use preterm birth as an outcome, gestational age of $<280 \mathrm{~d}$ was evaluated. The mothers in the highest physical load category had a higher risk (OR 1.4) than those in the lowest category and the mothers whose short-term physical load was high were also at higher risk (OR 1.2). When the analyses were restricted to women in the two lower socioeconomic classes, this increased risk was no longer apparent.

Launer and her colleagues (30) used an activity score based on type of work, sitting or standing position, physical intensity level, and number of hours of work to assess the relationship between physical demands and preterm birth in a prospective study of 15786 Guatemalan women. The investigators also made a unique attempt to evaluate factors that might modify physical demands, such as the number of children at home and the amount of help the subjects had with housework. These factors, however, were not examined to see if they modified the effect of work-related physical activity on outcome. After adjustment for monthly income and maternal weight and height, working in a standing position was associated with an increased risk of preterm birth (OR 1.6), and working in a walking position was associated with a twofold risk of preterm babies small for their gestational age. The activity score was linearly related to the proportion of preterm babies small for their gestational age, women in the highest demand category having the highest proportion. This same trend was apparent but not statistically significant for preterm birth.

The question remains of whether investigators have succeeded in truly disentangling the effects of socioeconomic status from the effects of ergonomic stressors. In the studies discussed previously that have examined the association between physically demanding work and preterm birth within socioeconomic strata $(26,27,28)$, even the study by McDonald et al (28) did not have sufficient women in the higher socioeconomic stratum with ergonomically stressful work to assess the relationship within that stratum. The study reported an association between heavy lifting and pre- term birth in all occupational sectors, although only the sectors representing lower socioeconomic status had sufficient numbers individually to reach statistical significance. In the two studies by Saurel-Cubizolles and her colleagues $(26,27)$, the relationship between ergonomically stressful work and preterm birth was apparent in the lower socioeconomic stratum, but the number of women with ergonomically stressful work in the higher stratum was too small to permit any evaluation.

Recognizing the inherent difficulty of disentangling socioeconomic status and ergonomically stressful work, Klebanoff and his co-workers (31) selected a study population of uniformly high socioeconomic status (989 female medical residents and, as a comparison, 1238 wives of male residents). Questionnaires were sent to all of the women and a random sample of men who graduated from medical school in 1985 . The questions were asked about all pregnancies, in particular the first pregnancy to begin during residency, and about certain aspects of the residency. Although the only individual physical stressors considered were number of hours worked per week and the number of nights on call, residency is generally characterized by prolonged standing, long hours, and stress. The investigators reported that there were no differences in the proportion of preterm births between the female residents and residents' wives. The women in surgical specialties were at higher risk (OR 1.6) than the other residents, although this risk was not statistically significant. The risk of preterm birth was twice as high for the residents who worked $100 \mathrm{~h}$ a week than for those who worked less. The investigators concluded that working long hours (as long as it was less than $100 \mathrm{~h}$ ) in a stressful occupation did not have an effect on the outcome of pregnancy in a healthy, higher socioeconomic status population.

However, the investigators also found that female residents were twice as likely to experience premature labor as wives of male residents. To explain this finding, they suggested that attending obstetricians might be more likely to diagnose early labor in a group of women perceived to be at high risk, that female residents may be more likely to report symptoms, or that preterm labor due to work-related stress could be more reversible. However, a likely alternative explanation is that residents who go into premature labor are treated earlier and more aggressively, and therefore more successfully. Thus, while their conclusion that women medical residents are not at increased risk for premature birth is supported by their data, the results may not be generalizable to women in other stressful occupations with less access to early diagnosis and treatment.

In summary, while no individual study is persuasive regarding the association of ergonomically stressful work and premature delivery, the literature as a whole suggests that such an association exists. Whether this association varies by social class is not yet clear. 
Resolving this question would undoubtedly provide scientific insight into the underlying nature of the association, and thus a resolution is worth pursuing. For public health the question is less pressing, inasmuch as most of the patients who are engaged in ergonomically stressful work are also of lower socioeconomic status.

The utility of the cumulative fatigue score proposed by Mamelle and her colleagues also needs more investigation. For epidemiologic studies of the relationship between ergonomic stressors and pregnancy outcomes, it appears to be inadequate. Divergent aspects of work are pooled together in a single source, each source is assumed to have an independent and additive effect, and joint effects between various sources cannot be examined (32). In short, use of the scoring system does not permit the relative contributions of each source to be defined, and thus the identification of the priorities for possible job modifications during pregnancy is not possible. These difficulties are compounded by vagueness of the definition of many of the sources. However, the question remains of whether such a scoring system could be clinically useful in identifying women at high risk of preterm birth. Mamelle \& Munoz have also urged that the primary use of the occupational fatigue index is as a predictor of premature birth rather than as an epidemiologic tool (33). However, its utility in this regard still remains to be demonstrated.

\section{Effects on birthweight}

In contrast to the data on premature delivery, the data on birthweight are more contradictory. As with premature delivery, no individual ergonomic stressor has been consistently found to be associated with effects on birthweight, although studies examining specific stressors are few (table 3). Six studies have used a composite score or some other assessment of overall activity level. Three of them have found an effect on birthweight and three have not.

Of the three studies that found an association between physically stressful work and birthweight (27, 29,30 ), one (27) did not control for the effect of gestational age. Since this study also found a strong association between the composite score and preterm birth, it is not possible to judge the extent to which the decrease in birthweight was secondary to the decrease in gestational age. Launer and her co-workers' prospective study (30) of Guatemalan women found that women who worked in a standing or walking position were at higher risk of having babies that were small for their gestational age (OR 1.2 and OR 1.3, respectively) and that there was a direct relationship between a physical activity score and the proportion of babies small for their gestational age and those both small for their gestational age and preterm. Although the study appears to be methodologically sound, the described nutritional status of the study population ap- pears to be poorer than that of populations in most developed countries. In addition, the definition of "small for gestational age" was based on norms from a well-nourished population, and fully $20 \%$ of the women gave birth to babies meeting this definition. It is possible that physically stressful work has a more deleterious effect on women with little nutritional reserve. Thus, while the conclusions of the study may well be valid for the study population, they are not necessarily generalizable to developed countries.

The third study reporting an association between decreased birthweight and physically stressful work, that by Nurminen and her co-workers (29), found an increased risk of babies small for their gestational age among women in the highest mean (OR 2.4) and highest short-term (OR 1.8) physical work load categories, when the analyses were restricted to women in nonagricultural, lower socioeconomic categories. However, the association was not as strong overall. There was only a $55-\mathrm{g}$ difference in the median birthweight between the highest and lowest work load categories.

This weak, but positive, evidence of an association between birthweight and physical exertion has not been confirmed in other studies. Rabkin et al (34) conducted a prospective study of 1507 women, of whom 626 worked more than $30 \mathrm{~h}$ a week and 202 worked parttime. In addition to a questionnaire at the initial prenatal visit, follow-up questionnaires were administered at 17,28 , and 36 weeks. An activity measure, based on the activity of the day before the interview, considered the nature of paid employment, the usual posture at work, the starting and stopping time, commuting time and mode, amount of time spent performing child care and housework, and the nature and duration of leisure activities. Energy requirements in kilocalories per minute were estimated, and the value was used as one of the measures of exposure. Overall, no association was found between mean birthweight and any measure of exposure, including hours of paid work, posture at work, or energy expenditure. Although the study was relatively small, it had sufficient power to detect an 80-g difference in birthweight. And, while the calculation of energy expenditure involved several assumptions that could have led to misclassification of exposure, there was no such possibility for the other measures, such as hours worked and posture.

While the initial report from the Montreal survey (28) found an association between birthweight and several ergonomic stressors, the effect of gestational age was not considered. A further analysis (35) in which birthweight was controlled for gestational age confirmed the association between birthweight and both lifting heavy weights and shift work, but the association with working more than $45 \mathrm{~h}$ a week and the occupational fatigue index was no longer apparent. The large size of the study population and its comprehensive assessment of ergonomic exposures lend weight to the reported findings, both positive and negative. 
The study by Klebanoff and his colleagues (31), although it did not contain an explicit measure of physical exertion, is the third important study that did not find an association between physically strenuous work and birthweight. In their comparison of the pregnancy outcomes of female medical residents and wives of male medical residents, there were no differences in the proportion of women delivering infants small for their gestational age or babies weighing less than 2500 or $1500 \mathrm{~g}$ at birth or in the mean birthweight of the babies. There was also no difference in the proportion of babies small for their gestational age between residents who worked more than $100 \mathrm{~h}$ a week and those who worked less.

Thus, in contrast to the relatively consistent data between a cumulative effect of ergonomic exposures and preterm delivery, the evidence for an association with birthweight is both limited and variable. However, such an association cannot be ruled out. There is evidence that physically stressful work has an adverse impact on birthweight in less developed countries (eg, reference 36 ), and this association is biologically plausible. Nonetheless, the weight of the evidence suggests that any adverse effect of ergonomic stressors on birthweight in industrialized countries, if it exists, is likely to be small.

\section{Summary and recommendations}

The need for more research on the effect of ergonomic stressors is clear. In epidemiologic research, the greatest challenge is a more precise description and quantification of ergonomic exposures. This challenge must be addressed on several fronts. Questionnaires need to be refined and their reliability assessed; the validation of questionnaires, while a difficult task, would also be extremely useful; newer methods that evaluate energy expenditure directly and are applicable for large studies must be developed; and the effects of ergonomic stressors encountered outside of the workplace also need to be incorporated into future studies. In addition, further attempts to explore the roles of psychological stress and socioeconomic status as confounders and effect modifiers are necessary.

In addition to epidemiologic research, experimental research in which physical activity can be carefully quantified should also help clarify the impact of ergonomic exposures. For example, a recent Massachusetts study investigated the frequency of uterine contractions using ambulatory tocodynamometry before, during, and after standardized work activities performed by low risk women between 28 and 32 weeks of gestation (M Paul, personal communication). Such research will help quantify the effect of ergonomic exposures and provide a scientific basis for rational intervention.

Nonetheless, sufficient scientific evidence exists to support certain recommendations. Clinicians caring for pregnant working women must elicit in some detail the number and type of ergonomic stressors to which their patient is exposed. Simply knowing the job title is insufficient, as jobs with the same title can vary widely in their exposures. Questions should be directed toward obtaining adequate data on the number of hours worked, the number of hours spent standing, the frequency of lifting and the approximate weight lifted, the woman's perception of the physical strenuousness of her job, work on a machine, and the nature of the work environment. The clinician's assessment of the extent to which a woman's exposure to ergonomic stressors places her at increased risk for preterm birth will depend on the nature and number of ergonomic stressors her job entails, the presence or absence of other risk factors, and her health status. Preventive intervention can range from a discussion of the possible risk to job modifications or transfer to intensive surveillance of the pregnancy to leaving work. The available evidence does not suggest that a blanket mandatory leave policy would be useful. It does, however, provide support for any decisions involving the mitigation of ergonomic stressors made by a woman and her health practitioner.

\section{Acknowledgments}

This work was aided by grant $15-126$ (Reproductive Hazards in the Workplace, Home, Community, and Environment) from the March of Dimes Birth Defects Foundation.

\section{References}

1. Fuchs F, Stubblefield PG. Preterm birth: causes, prevention, and management. New York, NY: McMillan Publishers, 1984.

2. Goulet L, Thériault G. Association between spontaneous abortion and ergonomic factors: a literature review of the epidemiologic evidence. Scand J Work Environ Health 1987;13:399-403.

3. World Health Organization (WHO). Manual of the international statistical classification of diseases, injuries and causes of death; vol 1 (9th revision). Geneva: WHO, 1975.

4. Alberman E. Low birthweight. In: Bracken MB, ed. Perinatal epidemiology. New York, NY: Oxford University Press, 1984:86-98.

5. Van den Berg BJ, Oechsli FW. Prematurity. In: Bracken MB, ed. Perinatal epidemiology. New York, NY: Oxford University Press, 1984:69-85.

6. Marbury MC, Linn S, Monson RR, Wegman DH, Stubblefield PG, Ryan KJ. Work and pregnancy. J Occup Med 1984;26(6):415-21.

7. Douglas JWB. Some factors associated with prematurity. J Obstet Gynaecol Br Emp 1950;57:143-70.

8. Stewart AM. A note on the obstetric effects of work during pregnancy. Br J Prev Soc Med 1955;9:159.

9. Illsley R, Billewicz WZ, Thomson AM. Prematurity and paid work during pregnancy. Br J Prev Soc Med 1954; 8:153.

10. Gofin $\mathbf{J}$. The effect on birthweight of employment during pregnancy. J Biosoc Sci 1979;11:259.

11. Saurel MN, Kaminski M. Pregnant women at work. 
Lancet $1983 ; 1: 475$.

12. Murphy JF, Dauncey M, Newcombe R. Employment in pregnancy: prevalence, maternal characteristics, perinatal outcome. Lancet 1984;2:1163-8.

13. Najman JM, Morrison J, Williams GM, Andersen MJ, Keeping JD. The employment of mothers and the outcomes of their pregnancies: an Australian study. Public Health 1989;103:189-98.

14. Zuckerman BS, Frank DA, Hingson R, Morelock S, Kayne HL. Impact of maternal work outside the home during pregnancy on neonatal outcome. Pediatrics 1986; $77(4): 459-64$.

15. Naeye RL, Peters EC. Working during pregnancy: effects on the fetus. Pediatrics 1982;69:724-7.

16. Manshade JP, Eeckels R, Manshade-Desmet V, Vlietinck R. Rest versus heavy work during the last weeks of pregnancy: influence on fetal growth. $\mathrm{Br} \mathrm{J}$ Obstet Gynaecol 1987;94:1059-67.

17. Berkowitz GS, Kelsey JL, Holford TR, Berkowitz RL. Physical activity and the risk of spontaneous preterm delivery. J Reprod Med 1983;28(9):581-8.

18. Hartikainen-Sorri A-L, Sorri M. Occupational and socio-medical factors in preterm birth. Obstet Gynecol 1989;74:13-6.

19. Ahlborg G, Bodin L, Hogstedt C. Heavy lifting during pregnancy - a hazard to the fetus?: a prospective study. Int J Epidemiol 1990;19(1):90-7.

20. Meyer BA, Daling JR. Activity level of mother's usual occupation and low infant birth weight. J Occup Med 1985;27(11):841-7.

21. Teitelman AM, Welch LS, Hellenbrand KG, Bracken MB. Effect of maternal work activity on preterm birth and low birth weight. Am J Epidemiol 1990;131(1): 104-13.

22. Homer CJ, Beresford SAA, James SA, Siegel E, Wilcox S. Work-related physical exertion and risk of preterm, low birthweight delivery. Paediatr Perinatal Epidemiol 1990;4:161-74.

23. Ramirez G, Grimes RM, Annegers JF, Davis BR, Slater $\mathrm{CH}$. Occupational physical activity and other risk factors for preterm birth among US army primigravidas. Am J Public Health 1990;80(6):728-30.

24. Mamelle N, Laumon B, Lazar P. Prematurity and occupational activity during pregnancy. Am J Epidemiol 1984;119(3):309-22.
25. Mamelle N, Munoz F. Occupational working conditions and preterm birth: a reliable scoring system. Am J Epidemiol 1987;126(1):150-2.

26. Saurel-Cubizolles MJ, Kaminski M, Llado-Arkhipoff J, Mazabrun CD, Estryn-Behar M, Berthier C, et al, Pregnancy and its outcome among hospital personnel according to occupation and working conditions. J Epidemiol Community Health 1985;39:129-34.

27. Saurel-Cubizolles MJ, Kaminski M. Pregnant women's working conditions and their changes during pregnancy: a national study in France. Br J Ind Med 1987;44: 236-43.

28. McDonald AD, McDonald JC, Armstrong B, Cherry NM, Nolin AD. Prematurity and work in pregnancy. Br J Ind Med 1988;45:56-62.

29. Nurminen T, Lusa S, Ilmarinen J, Kurppa K. Physical work load, fetal development and course of pregnancy. Scand J Work Environ Health 1989;15:404-14.

30. Launer LJ, Villar J, Kestler E, De Onis M. The effect of maternal work on fetal growth and duration of pregnancy: a prospective study. Br J Obstet Gynaecol 1990; 97:62-70.

31. Klebanoff MA, Shiono PH, Rhoads GG. Outcomes of pregnancy in a national sample of resident physicians. N Engl J Med 1990;323:1040-5.

32. Punnett L, Marbury M. Re: "Occupational working conditions and preterm birth: a reliable scoring system" [Letter to the editor]. Am J Epidemiol 1989;129(2):451.

33. Mamelle N, Munoz F. Re: 'Occupational working conditions and preterm birth: the authors reply" [Letter to the editor]. Am J Epidemiol 1989;129(2):451.

34. Rabkin CS, Anderson HR, Bland JM, Brooke OG, Chamberlain G, Peacock JL. Maternal activity and birth weight: a prospective population-based study. Am J Epidemiol 1990;131(3):522-31.

35. Armstrong BG, Nolin AD, McDonald AD. Work in pregnancy and birth weight for gestational age. $\mathrm{Br} \mathrm{J}$ Ind Med 1989;46:196-9.

36. Tafari N, Naeye RL, Gobezie A. Effects of maternal undernutrition and heavy physical work during pregnancy on birth weight. Br J Obstet Gynecol 1980;87: $222-6$.

Received for publication: 22 July 1991 